\title{
Validation of a scoring system to evaluate the risk of rapid growth of intracranial meningiomas in neurofibromatosis type 2 patients
}

\author{
Samiya Abi Jaoude, MD, ${ }^{1}$ Matthieu Peyre, MD, PhD, ${ }^{1,2}$ Vincent Degos, MD, PhD, ,,3 \\ Stéphane Goutagny, MD, PhD, ${ }^{4}$ Béatrice Parfait, $\mathrm{PhD},{ }^{5}$ and Michel Kalamarides, MD, $\mathrm{PhD}{ }^{1,2}$ \\ 1Department of Neurosurgery and ${ }^{3}$ Neurosurgical Intensive Care, Pitié-Salpêtrière Hospital, Assistance Publique-Hôpitaux de \\ Paris; ${ }^{2}$ Sorbonne Universités, Paris; ${ }^{4}$ Department of Neurosurgery, Beaujon Hospital, Assistance Publique-Hôpitaux de Paris; \\ and ${ }^{5}$ Department of Genetics and Molecular Biology, Cochin Hospital, Assistance Publique-Hôpitaux de Paris, France
}

\begin{abstract}
OBJECTIVE Intracranial meningiomas occur in about half of neurofibromatosis type 2 (NF2) patients and are very frequently multiple. Thus, estimating individual meningiomas' growth rates is of great interest to tailor therapeutic interventions. The Asan Intracranial Meningioma Scoring System (AIMSS) has recently been published to estimate the risk of tumor growth in sporadic meningiomas. The current study aimed to determine predictors of rapid meningioma growth in NF2 patients and to evaluate the AIMSS score in a specific NF2 cohort.

METHODS The authors performed a retrospective analysis of 92 NF2 patients with 358 measured intracranial meningiomas that had been observed prospectively between 2012 and 2018. Tumor volumes were measured at diagnosis and at each follow-up visit. The growth rates were determined and evaluated with respect to the clinicoradiological parameters. Predictors of rapid tumor growth (defined as growth $\geq 2 \mathrm{~cm}^{3} / \mathrm{yr}$ ) were analyzed using univariate followed by multivariate logistic regression to build a dedicated predicting model. Receiver operating characteristic $(R O C)$ curves to predict the risk of rapid tumor growth with the AIMSS versus the authors' multivariate model were compared.

RESULTS Sixty tumors (16.76\%) showed rapid growth. After multivariate analysis, a larger tumor volume at diagnosis $(p<0.0001)$, presence of peritumoral edema $(p=0.022)$, absence of calcifications $(p<0.0001)$, and hyperintense or isointense signal on T2-weighted MRI $(p<0.005)$ were statistically significantly associated with rapid tumor growth. It is particularly notable that the genetic severity score did not seem to influence the growth rate of NF2 meningiomas. In comparison with the AIMSS, the authors' multivariate model's prediction did not show a statistically significant difference (area under the curve [AUC] 0.82 [95\% $\mathrm{Cl} 0.76-0.88$ ] for the AIMSS vs AUC 0.86 [95\% $\mathrm{Cl} 0.81-0.91]$ for the authors' model, $p=0.1$ ).
\end{abstract}

CONCLUSIONS The AIMSS score is valid in the authors' cohort of NF2-related meningiomas. It adequately predicted risk of rapid meningioma growth and could aid in decision-making in NF2 patients.

https://thejns.org/doi/abs/10.3171/2020.3.JNS192382

KEYWORDS neurofibromatosis type 2; meningioma; natural history; risk assessment; oncology

$\mathrm{N}$ EUROFIBROMATOSIS type 2 (NF2) is a rare genetic disorder characterized by the development of multiple benign tumors of the nervous system. ${ }^{1,2}$ Its hallmark is bilateral vestibular schwannomas. ${ }^{3}$ Intracranial meningiomas are the second most common intracranial tumors in NF2, occurring in $45 \%-58 \%$ of patients; they are often multiple, cause significant morbidity, ${ }^{2}$ and are a leading cause of mortality. ${ }^{4,5} \mathrm{NF} 2$ patients with intracranial meningiomas most often present with a severe phenotype of the disease $\mathrm{e}^{4}$ and have an increased relative risk of mortality (RR 2.51) compared with those without meningiomas. ${ }^{6}$

Management of NF2-associated meningiomas is based on observation, radiosurgery/radiotherapy, and surgery, with surgery being the main treatment option in the case of significantly growing and/or symptomatic tumors. Multiple surgical procedures are often necessary during the lifetime of an NF2 patient due to a high tumor burden. One

ABBREVIATIONS AIMSS = Asan Intracranial Meningioma Scoring System; AUC = area under the curve; GKRS = Gamma Knife radiosurgery; NF2 = neurofibromatosis type 2; ROC = receiver operating characteristic; $\mathrm{T} 2 \mathrm{~W}=\mathrm{T} 2$-weighted.

SUBMITTED August 31, 2019. ACCEPTED March 17, 2020.

INCLUDE WHEN CITING Published online May 22, 2020; DOI: 10.3171/2020.3.JNS192382. 
of the challenges of managing NF2 patients with multiple meningiomas is therefore to proactively choose which tumor to treat, allowing for an ideal benefit/risk balance. In order to do so, estimating which meningiomas are most likely to grow rapidly is of great interest, particularly for smaller meningiomas potentially accessible to radiosurgery.

Predicting the growth of meningiomas remains nonetheless challenging. Only a few studies have identified factors that might be associated with increased tumor growth of NF2-related meningiomas: ${ }^{7,8}$ younger age at onset, female sex, and tumor location, but they relied on a small number of patients. As multiple meningiomas appear to behave differently within the same individual, ${ }^{7,8}$ future studies should focus on tumor-related rather than patientrelated prognostic factors. The use of tumor growth patterns may also be difficult. While most meningiomas in NF2 patients demonstrate no or minimal growth on serial imaging, up to $10 \%$ demonstrate rapid growth. ${ }^{5}$ Those growing meningiomas may display linear, exponential, or saltatory growth patterns, ${ }^{7-9}$ similarly to sporadic tumors..$^{10}$ Those growth patterns are difficult to predict.

Interestingly, a recent study by Lee et al. presented a novel scoring system for estimating rapid growth in untreated asymptomatic sporadic meningiomas based on tumor size, brain edema, calcifications, and signal on T2weighted (T2W) MRI. ${ }^{11}$ The Asan Intracranial Meningioma Scoring System (AIMSS) yielded reproducible results and a good predictive value.

The objectives of this study were to describe the natural history of meningiomas in a large series of NF2 patients, to determine predictors of rapid tumor growth, and to assess the reproducibility of the AIMSS in this specific population.

\section{Methods \\ Patient Population}

Between 2012 and 2018, 230 patients with diagnosed NF2 were routinely followed up at our national NF2 referral center with an annual clinical workup, including audiometry, cranial and spinal MRI, otology and neurosurgery consultations, speech therapy, and psychological interview. Diagnosis of NF2 was based on the Manchester (modified National Institutes of Health) diagnostic criteria., ${ }^{1,12}$ Data were obtained retrospectively from clinical records and copies of imaging studies. All living patients provided informed written consent, and the local institutional review committee approved the retrospective chart review.

A total of 113 patients (49.13\%) harbored at least 1 intracranial meningioma. Patients with a follow-up duration $\leq 2$ years $(n=9)$, those who did not have $>2$ imaging modalities more than 2 years apart $(n=10)$, and those whose files were incomplete $(n=2)$ were excluded. Thus, the final number of patients included in the study was 92 .

Meningiomas were diagnosed and followed up with neuroimaging analysis of contrast-enhanced T1-weighted MRI. Occasionally, some patients were followed up with CT studies with contrast when they had a cochlear or auditory brainstem implant. All digital imaging studies performed outside our hospitals' network were imported into the local PACS Carestream network using the Data Import tool.

The 92 patients harbored a total of 517 meningiomas. We aimed to measure the maximum number of meningiomas for which we could obtain good-quality images and complete radiological files during our retrospective collection of data. We excluded 34 operated tumors for which we could not retrieve the images obtained at the time of surgery. A further 30 meningiomas could not be measured on follow-up because the patients had benefited during follow-up from cochlear or auditory brainstem implants ipsilateral to the meningiomas, resulting in distorted images on MRI and CT scans. When a patient had multiple nodules along the convexity and the falx, only meningiomas that could clearly be visually delineated from the rest were measured. The total number of meningiomas included was 358.

\section{MRI Assessment and Determination of the Tumor Growth Rate}

All tumor volumes were measured on enhanced imaging series by the same author (S.A.J.) to minimize interuser variability using the inherent tools of the PACS Carestream technology. Tumor volumes were obtained through manual segmentation and/or semiautomatic segmentation with systematic review and correction using the Livewire correction tool for every slice. All imaging studies available for every patient were measured. The volumes included in the final database were the volume at the time of first available investigation, which was considered the baseline, volume at any resection or radiotherapy, postoperative volume, and volume at last follow-up.

The absolute growth rate $\left(\mathrm{cm}^{3} / \mathrm{yr}\right)$ was calculated using the following formula: (latest tumor size in $\mathrm{cm}^{3}$ - initial tumor size in $\mathrm{cm}^{3}$ )/interval of follow-up in years. We used the rate of $2 \mathrm{~cm}^{3} / \mathrm{yr}$ as a cutoff to categorize the meningiomas into either the slow-growth group $\left(<2 \mathrm{~cm}^{3} / \mathrm{yr}\right)$ or the rapid-growth group $\left(\geq 2 \mathrm{~cm}^{3} / \mathrm{yr}\right)$ as per the criteria used in the article describing the AIMSS score by Lee et al. ${ }^{11}$ Since our aim was to assess the reproducibility of the AIMSS in the NF2 population, we decided to use the same criterion of an annual growth rate of $\geq 2 \mathrm{~cm}^{3} / \mathrm{yr}$ as the criterion defining rapid growth. The relative growth rate was calculated based on the assumption that tumors grow geometrically using the following formula: linear relative growth rate $(\% / y r)=($ absolute growth rate/initial tumor size in $\left.\mathrm{cm}^{3}\right) \times 100$.

We then evaluated growth rate with respect to the follow-up clinicoradiological parameters: age, sex, genetic severity score, ${ }^{13-15}$ NF2 phenotype according to Parry et al., ${ }^{16}$ age at diagnosis of meningiomas, tumor load (unique vs multiple), tumor location, presence of neurological symptoms, calcifications, peritumoral edema, signal intensity on T2W MRI, and initial tumor volume. When a tumor had undergone surgery, we also evaluated the growth rate with regard to histopathological grade.

\section{The AIMSS}

The AIMSS score is a recently developed weighted 
scoring system that estimates the risk of rapid meningioma growth based on the patient's clinicoradiological characteristics. ${ }^{11}$ The AIMSS parameters are tumor size, calcifications, peritumoral edema, and signal intensity on T2W MRI. The risk of rapid tumor growth according to the AIMSS score is estimated to be $<10 \%$ when the total score is $0-2,10 \%-50 \%$ when the total score is $3-6$, and $>50 \%$ when the total score is $7-11$. The scoring system is shown in Table 1.

\section{Statistical Analysis}

Statistical analyses were performed in Stata software (StataCorp LLC); chi-square tests or Fisher exact tests were used for categorical variables and the Mann-Whitney U-test (2-tailed) for continuous unpaired variables. The databases were pooled by patient for the demographic characteristics and by meningioma for the statistical analyses, as our objective aims to identify predictors of rapid growth for every meningioma in the NF2 population. Values are expressed as mean \pm SD. Statistical significance was presumed at $\mathrm{p}<0.05$. Predictors of rapid tumor growth were analyzed with univariate followed by multivariate logistic regression to build a dedicated predicting model. Receiver operating characteristic (ROC) curves to predict the risk of rapid tumor growth with the AIMSS versus our multivariate model were compared. A Cochran-Armitage trend test was also used first with the asymptotic $\mathrm{p}$ value and then with the Monte Carlo method to assess association between growth rates and the genetic severity score.

\section{Results \\ Patient Demographics}

The 92 NF2 patients included in this study harbored a total of 517 intracranial meningiomas. Sixteen patients $(17.39 \%)$ had 1 meningioma and $76(82.61 \%)$ had $\geq 2$ meningiomas; 17 patients $(18.48 \%)$ had $\geq 10$ meningiomas. Meningiomas were more common in female patients: 55 female patients $(59.78 \%)$ and 37 male patients $(40.22 \%)$. The mean duration of follow-up after NF2 diagnosis was 186 months (24-432 months). There was no statistical significance between age or sex and tumor growth rates.

\section{Natural History of Intracranial Meningiomas}

The total number of meningiomas included in our study was 358. The tumor characteristics are summarized in Table 2. The intracranial meningiomas in our cohort were more frequently located at the convexity and along the falx $(\mathrm{n}=255,71.23 \%)$, but location was not correlated with growth rate. Most meningiomas (83.52\%) in NF2 patients were asymptomatic. The most common symptoms were partial seizures (18 patients), intracranial hypertension (12 patients), motor deficit (10 patients), and cranial nerve palsy ( 9 patients).

The mean tumor volume at diagnosis was $5.43 \pm 12.42$ $\mathrm{cm}^{3}$. At presentation, fast-growing meningiomas were significantly larger $\left(14.71 \pm 21.6 \mathrm{~cm}^{3}\right)$ than slow-growing meningiomas $\left(2.26 \pm 3.83 \mathrm{~cm}^{3}\right)(\mathrm{p}<0.0001)$. While a larger initial volume could be a potential bias, this was not the case in our series. To determine the relationship between
TABLE 1. AIMSS

\begin{tabular}{cc}
\hline Category & Score \\
\hline Tumor diameter, cm & \\
\hline$\leq 2.5$ & 0 \\
$>2.5$ to $\leq 4.0$ & 3 \\
\hline$>4.0$ & 6 \\
\hline Calcifications & 0 \\
\hline Present & 2 \\
\hline Absent & \\
\hline Peritumoral edema & 1 \\
\hline Present & 0 \\
\hline Absent & 0 \\
\hline T2W MRI & 2 \\
\hline Hypointense & \\
\hline Hyperintense/isointense &
\end{tabular}

Distilled from the AIMSS scoring system of Lee et al.11

tumor size and growth rate, we correlated the absolute growth rates to the initial tumor diameters. As depicted in Supplemental Fig. 1, most tumors with a smaller initial volume have a slower growth rate (280/314), whereas larger tumors (initial diameter $>4 \mathrm{~cm}$ ) all present with rapid growth. In the rapid-growth group, the annual growth rates increased in proportion to the diameter with a mean slope of $1.8 \mathrm{~cm}^{3} / \mathrm{yr} /$ diameter $(\mathrm{cm})$, whereas those in the slow-growth group were almost stationary, regardless of the diameter increase (Supplemental Fig. 2). The mean annual growth rate for the entire cohort was $2.24 \pm 7.24 \mathrm{~cm}^{3} /$ yr. The mean growth rate for slow-growing meningiomas was $0.413 \pm 4.95 \mathrm{~cm}^{3} / \mathrm{yr}$, while that of fast-growing meningiomas was $9.66 \pm 13.16 \mathrm{~cm}^{3} / \mathrm{yr}$.

Of the 358 measured meningiomas, 298 (83.24\%) had a slow-growth pattern, including 94 with no growth, 165 with an annual growth of 0.1 to $1 \mathrm{~cm}^{3} / \mathrm{yr}$, and 39 with an annual growth of 1 to $2 \mathrm{~cm}^{3} / \mathrm{yr}$, whereas 60 meningiomas (16.76\%) had an annual growth rate of $\geq 2 \mathrm{~cm}^{3} / \mathrm{yr}$ (corresponding to a rapid growth). Meningioma growth rates are shown in Fig. 1.

Interestingly, multiple tumors in an individual patient follow different growth rates and do not increase in size simultaneously. In fact, all 60 rapid-growth tumors occurred in 37 of the 92 patients. Five of these patients had a single intracranial meningioma, and the remaining 32 patients had multiple meningiomas (32 patients with a total of 180 meningiomas). None of these 32 patients had the same pattern of growth for all of their meningiomas. There seems to be no correlation between the number of rapid-growth tumors and the number of tumors a patient has. We tested the hypothesis that growth rates of individual tumors within patients were correlated but did not show an association between tumor growth rates and individual patients (OR 1.007 [95\% CI 0.993-1.021], $\mathrm{p}=$ 0.33). As shown in Supplemental Fig. 3, we were not able to show that tumor growth patterns and tumor load were correlated. Patterns of growth differed widely among the meningiomas in the same patient; most of a patient's me- 
TABLE 2. Meningioma characteristics in the slow-growth and rapid-growth groups

\begin{tabular}{|c|c|c|c|c|}
\hline Characteristic & All & Slow Growth $(<2$ cm³/yr) & Rapid Growth ( $\left.\geq 2 \mathrm{~cm}^{3} / \mathrm{yr}\right)$ & p Value \\
\hline Total & 358 & $298(83.2)$ & $60(16.8)$ & \\
\hline Male sex & $103(28.8)$ & $76(25.5)$ & $27(45)$ & 0.001 \\
\hline \multicolumn{5}{|l|}{ Location } \\
\hline Skull base & $103(28.8)$ & $86(28.9)$ & $17(28.3)$ & \multirow{2}{*}{ NS } \\
\hline Convexity & $255(71.2)$ & $212(71.1)$ & $43(71.7)$ & \\
\hline Mean age at meningioma diagnosis, yrs & $27.5 \pm 12$ & $28 \pm 12$ & $22 \pm 9$ & NS \\
\hline \multicolumn{5}{|c|}{ Timing of meningioma diagnosis/diagnosis of NF2 } \\
\hline Present at NF2 diagnosis & $200(55.9)$ & $160(53.7)$ & $40(66.7)$ & \multirow{3}{*}{0.018} \\
\hline De novo & $138(38.5)$ & $119(39.9)$ & $19(31.7)$ & \\
\hline Unknown & $20(5.6)$ & $19(6.4)$ & $1(1.7)$ & \\
\hline \multicolumn{5}{|l|}{ Neurological symptoms } \\
\hline No & $299(83.5)$ & $268(89.9)$ & $31(51.7)$ & \multirow{3}{*}{$<0.0001$} \\
\hline Yes & $56(15.6)$ & $29(9.7)$ & $27(45)$ & \\
\hline Unknown & $3(0.8)$ & $1(0.3)$ & $2(3.3)$ & \\
\hline \multicolumn{5}{|l|}{ Therapeutic intervention } \\
\hline No & $290(81)$ & $269(90.3)$ & $21(35)$ & $<0.0001$ \\
\hline Yes & $68(18)$ & $29(9.7)$ & $39(65)$ & \multirow{4}{*}{$<0.0001$} \\
\hline Surgery & $66(18.4)$ & $27(9)$ & $39(65)$ & \\
\hline Surgery \& radiotherapy & $5(1.4)$ & $1(0.3)$ & $4(6.7)$ & \\
\hline GKRS & $2(0.6)$ & $2(0.7)$ & $0(0)$ & \\
\hline \multicolumn{5}{|c|}{ Pathological grading of the 66 resected meningiomas } \\
\hline Grade I & $25(37.9)$ & $14(56)$ & $11(44)$ & \multirow{2}{*}{0.031} \\
\hline Grade II & $26(39.4)$ & $8(30.8)$ & $18(69.2)$ & \\
\hline Grade III & $1(1.5)$ & $0(0)$ & $1(100)$ & NA \\
\hline Unknown & $14(21.2)$ & $5(35.7)$ & $9(64.3)$ & NA \\
\hline Presence of cerebral edema & $36(10.1)$ & $20(6.7)$ & $16(26.7)$ & $<0.0001$ \\
\hline Presence of calcifications & $97(27.1)$ & $88(29.5)$ & $9(15)$ & $<0.0001$ \\
\hline Hyper- or isointense signal on T2W MRI & $63(17.6)$ & $38(12.7)$ & $25(41.7)$ & $<0.0001$ \\
\hline Mean initial tumor vol, $\mathrm{cm}^{3}$ & $5.4 \pm 12.4$ & $2.3 \pm 3.8$ & $14.7 \pm 21.6$ & $<0.0001$ \\
\hline Mean annual growth rate, $\mathrm{cm}^{3} / \mathrm{yr}$ & $2.2 \pm 7.2$ & $0.4 \pm 4.9$ & $9.7 \pm 13.2$ & \\
\hline
\end{tabular}

$\mathrm{NA}=$ not applicable; $\mathrm{NS}=$ not significant.

Values represent the number of patients (\%) unless stated otherwise. Mean values are presented as mean \pm SD.

ningiomas would have slow growth and 1 or 2 meningiomas would have rapid growth and even then, the rapidly growing meningiomas would not grow at the same rate.

\section{Influence of NF2 Mutation}

Of the 92 patients with meningiomas, 77 underwent mutation testing. A germline pathogenic NF2 mutation was identified in 43 patients $(55.84 \%)$, including 2 mosaic mutations in blood. Somatic NF2 mutations were identified in tumor samples from 5 of the 34 patients whose blood screening results were negative. No genetic screening result was available for the remaining 15 patients.

Genetic severity was rated as severe, moderate, or mild. Truncating mutations in exons 1-13 in all cells were rated as severe. Moderate disease included 1) deletion not involving the promoter region or exon 1 ; 2) splice-site mutations in exons 1-8; and 3) mosaicism of truncating mutations in exons 1-13 in blood. Mild disease was classified as 1) a missense mutation or an in-frame deletion; 2) a large deletion involving the promoter region or exon $1 ; 3$ ) a splice-site mutation in exons 9-15; 4) mosaicism (excluding moderate criteria); and 5) no mutation identified on blood analysis. ${ }^{13-15}$

Thirty-seven patients $(40.22 \%)$ had at least 1 meningioma with a rapid growth rate $\left(\geq 2 \mathrm{~cm}^{3} / \mathrm{yr}\right)$. Among these patients, 20 had a mild genetic severity score; 3 , moderate; and 11 , severe $(p=0.37)$. These findings suggest that the genetic severity score does not influence the growth rate of NF2 patients' meningiomas.

\section{De Novo Meningiomas}

In our cohort, we noticed a high number of de novo meningiomas $(\mathrm{n}=138,38.55 \%)$, many arising in few patients. In fact, of the 92 patients included in this study, $21(22.83 \%)$ did not have any identifiable meningeal lesion on cerebral MRI done at the initial diagnosis of 


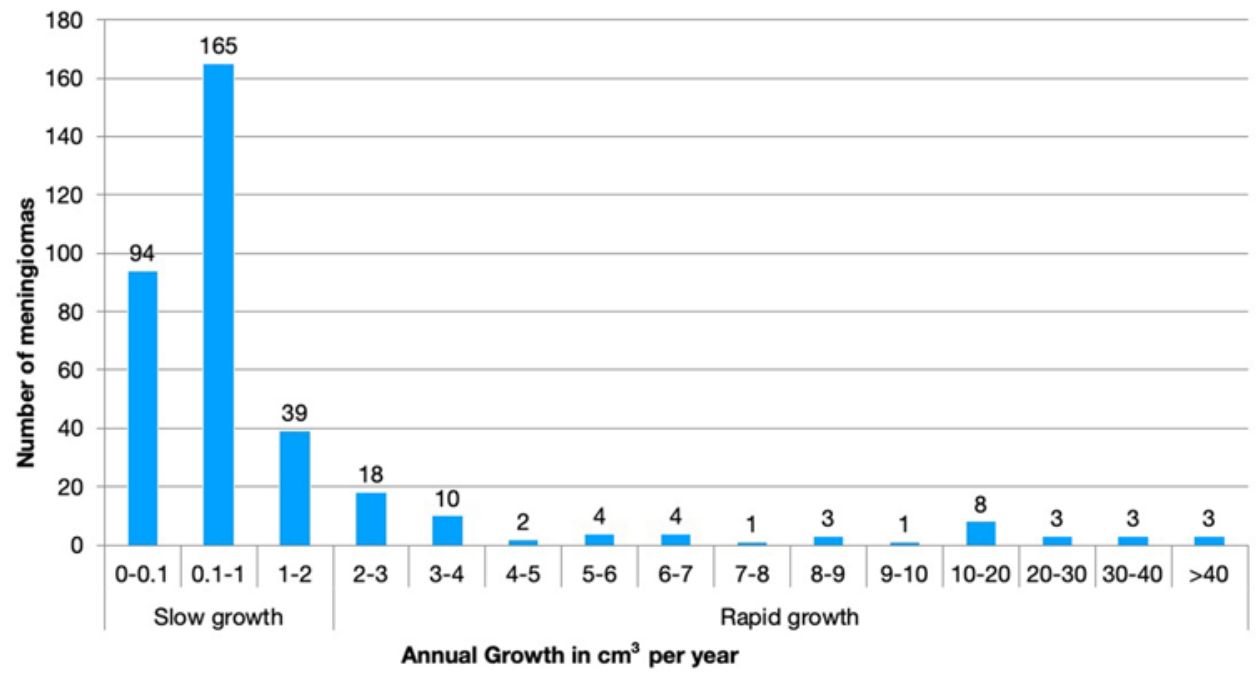

FIG. 1. Patterns of annual meningioma growth rates. Figure is available in color online only.

NF2. These de novo meningiomas had a behavior that was similar to those present at diagnosis of NF2 in terms of growth rates.

\section{Meningioma Surgery and Recurrence}

Of the 358 tumors, 66 were surgically treated; 25 were WHO grade I tumors, of which only 2 tumors recurred; 26 were grade II, of which 4 tumors recurred; and the only grade III tumor of the series (gross-total resection, with 28 mitotic figures in $10 \mathrm{hpf}$ and a Ki-67 of $15 \%$ ) has not recurred after 50 months of postoperative follow-up, and the patient has not received any adjuvant treatment postoperatively.

As expected, rapid-growth tumors were more likely to undergo surgery (39/60 rapid-growth tumors were operated on vs 27/298 slow-growth tumors, $\mathrm{p}<0.0001$ ). Unsurprisingly as well, most of the rapid-growth tumors that underwent surgery were grade II, while most of the slow-growth tumors were grade I $(p=0.031)$. Eleven of the rapid-growth resected tumors recurred compared with only 5 of the slow-growth resected tumors.

\section{Cerebral Edema}

Cerebral edema was present in $36(10.06 \%)$ of the 358 meningiomas. Tumors presenting with brain edema clearly had a more aggressive behavior: 18 of 36 were symptomatic, and 24 of 36 were surgically treated, of which 16 belonged to the rapid-growth group ( $<$ $0.0001)$. These tumors were significantly larger at diagnosis with a mean volume of $9.92 \pm 21.11 \mathrm{~cm}^{3}$ versus $3.6 \pm 8.01 \mathrm{~cm}^{3}(\mathrm{p}=0.0026)$, and they also had a higher growth rate per year $\left(7.24 \pm 15.67 \mathrm{~cm}^{3} / \mathrm{yr}\right)$ versus tumors presenting without edema $\left(1.46 \pm 5.10 \mathrm{~cm}^{3} / \mathrm{yr}\right)(\mathrm{p}=$ $0.0002)$. They required surgery more often $(24 / 36$, while only 42/322 meningiomas not associated with edema underwent surgery), and on pathological examination were more often grade II (17/24) than meningiomas not associated with brain edema, which were more often grade I $(21 / 42)(\mathrm{p}=0.00034)$.

\section{Calcifications}

Calcifications were present in 97 meningiomas, and they positively correlated with a slower growth pattern ( $\mathrm{p}$ $<0.0001$ ). At diagnosis, meningiomas with calcifications had a mean volume of $5.14 \pm 9.58 \mathrm{~cm}^{3}$ vs $3.89 \pm 10.45 \mathrm{~cm}^{3}$ $(p=0.0010)$. When a meningioma presented with calcifications, it was less likely to undergo surgery (only 10/97 intracranial meningiomas with calcifications were surgically treated, $\mathrm{p}=0.0145$ ). On pathological examination, these meningiomas were more often grade I (7/10), but the low number of calcified surgically treated meningiomas made this result not statistically significant.

\section{Signal Intensity on T2W MRI}

Sixty-three intracranial meningiomas had a hyperintense or isointense signal on T2W MRI, which positively correlated with a rapid growth rate $(\mathrm{p}<0.0001)$. At diagnosis, intracranial meningiomas with a hyper- or isointense signal on T2W MRI were significantly larger with a mean volume of $7.59 \pm 13.46 \mathrm{~cm}^{3}$ versus $3.53 \pm 9.41 \mathrm{~cm}^{3}$ $(\mathrm{p}=0.0002)$. Their growth rates were significantly higher $\left(5.54 \pm 12.76 \mathrm{~cm}^{3} / \mathrm{yr}\right.$ vs $\left.1.28 \pm 4.85 \mathrm{~cm}^{3} / \mathrm{yr}, \mathrm{p}<0.0001\right)$. When a meningioma had a hypointense signal on T2W MRI, it was less likely to undergo surgery $(p<0.0001)$. Signal on MRI had no predictive value on the histopathological grade.

\section{Reproducibility of the AIMSS in Meningiomas of NF2 Patients}

Our multivariate model's prediction did not show a statistically significant difference when compared with the AIMSS: our model's area under the curve (AUC) was 0.86 (95\% CI 0.81-0.91) versus AIMSS's AUC of 0.82 (95\% CI 0.76-0.88) $(\mathrm{p}=0.1)$. ROC curves are shown in Fig. 2.

The AIMSS score seems to be reproducible in NF2 patients: only $6 \%$ of the meningiomas classified by the AIMSS score as low risk had rapid growth, while 36\% of the intermediate-risk group and $93 \%$ of the high-risk meningiomas developed rapid exponential growth. These 


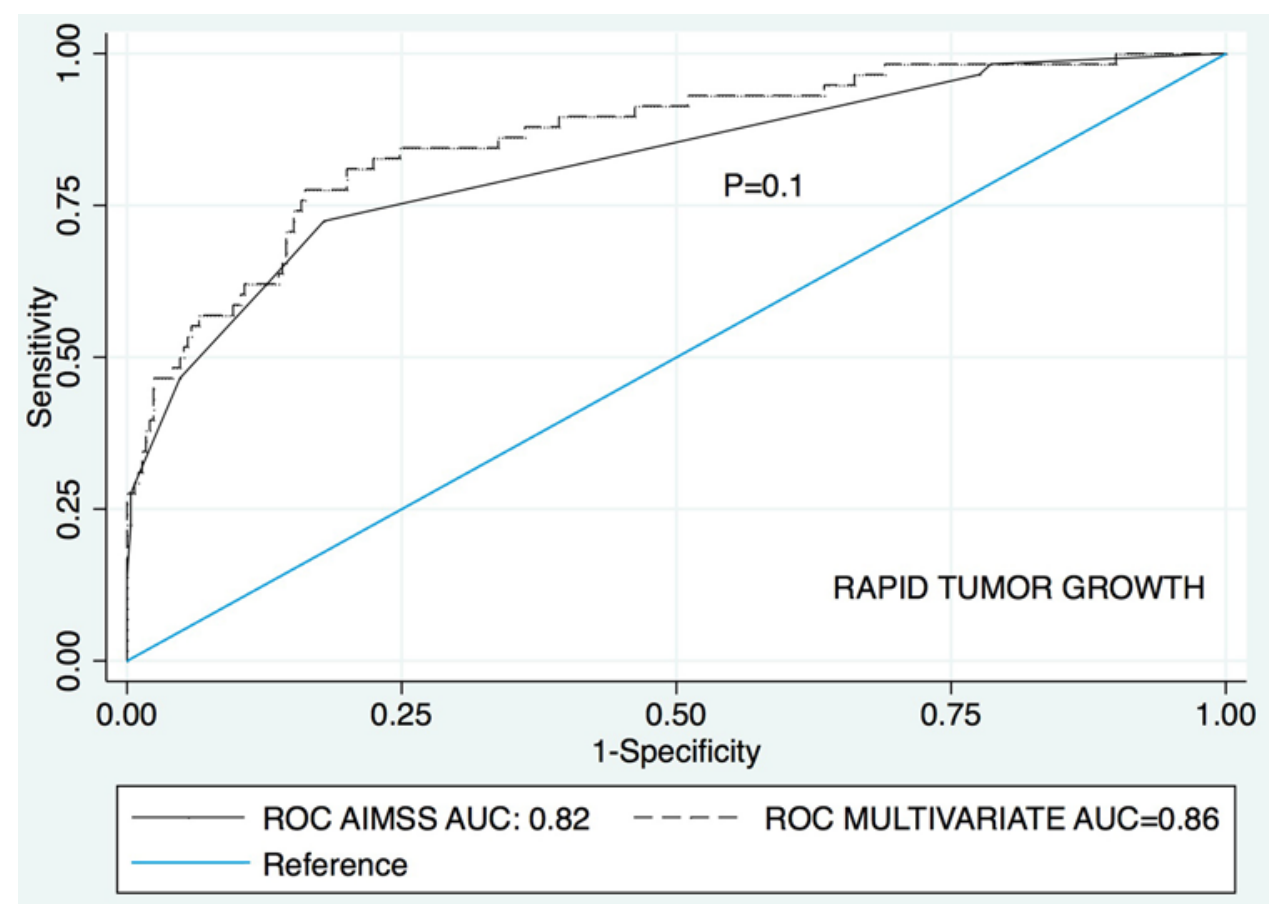

FIG. 2. Comparison of ROC curve of the AIMSS and that of our multivariate model. Our multivariate model's prediction did not show a statistically significant difference when compared with the AIMSS (AUC 0.86 [ $95 \% \mathrm{Cl} 0.81-0.91]$ for our model vs AUC $0.82[95 \% \mathrm{Cl} 0.76-0.88]$ for the AIMSS, $p=0.1)$. Figure is available in color online only.

results are summarized in Table 3 . Three examples of clinical applications of the AIMSS score to NF2 meningiomas are shown in Fig. 3.

\section{Discussion}

Identifying risk factors for the rapid growth of meningiomas in NF2 patients is paramount to deciding which tumors to treat and the correct timing of doing so. The risk factors identified in our study are a larger tumor volume at diagnosis, presence of peritumoral edema, absence of calcifications, and hyperintense or isointense signal on T2W MRI. These results seem in concordance with risk factors identified in the literature, ${ }^{4,5,11}$ which was reflected when we tested the AIMSS score in our population. The AIMSS score showed a good predictive value for rapid growth of meningiomas in NF2 patients. We were not able to construct a more robust predictive score for NF2 meningiomas with the use of variables such as age, sex, genetic severity score, NF2 phenotype according to Parry et al. ${ }^{16}$ age at diagnosis of meningioma, tumor load, tumor location, or presence of neurological symptoms.

TABLE 3. Probability of rapid tumor growth, estimated by the AIMSS and compared with observed value

\begin{tabular}{|c|c|c|c|c|}
\hline Score & Estimated Risk, \% & Observed Risk, $\%(n=358)^{*}$ & Observed Value by Risk Group, \% & Risk Group \\
\hline 0 & 2 & $1(1 / 67)$ & \multirow{3}{*}{6} & \multirow{3}{*}{$\begin{array}{l}\text { Low risk of rapid tumor growth } \\
(<10 \%)\end{array}$} \\
\hline 1 & 3 & $40(2 / 5)$ & & \\
\hline 2 & 6 & $7(14 / 188)$ & & \\
\hline 3 & 11 & $29(7 / 24)$ & \multirow{4}{*}{36} & \multirow{4}{*}{$\begin{array}{l}\text { Intermediate risk of rapid tumor } \\
\text { growth }(10-50 \%)\end{array}$} \\
\hline 4 & 19 & $27(9 / 33)$ & & \\
\hline 5 & 31 & $46(11 / 24)$ & & \\
\hline 6 & 47 & $100(3 / 3)$ & & \\
\hline 7 & 64 & $83(5 / 6)$ & \multirow{5}{*}{93} & \multirow{5}{*}{$\begin{array}{l}\text { High risk of rapid tumor growth } \\
(>50 \%)\end{array}$} \\
\hline 8 & 77 & $100(3 / 3)$ & & \\
\hline 9 & 87 & $100(1 / 1)$ & & \\
\hline 10 & 93 & $100(2 / 2)$ & & \\
\hline 11 & 96 & $100(2 / 2)$ & & \\
\hline
\end{tabular}

\footnotetext{
*Values represent the observed risk percentage (number of patients with rapid growth tumors/number of patients at each score).
} 

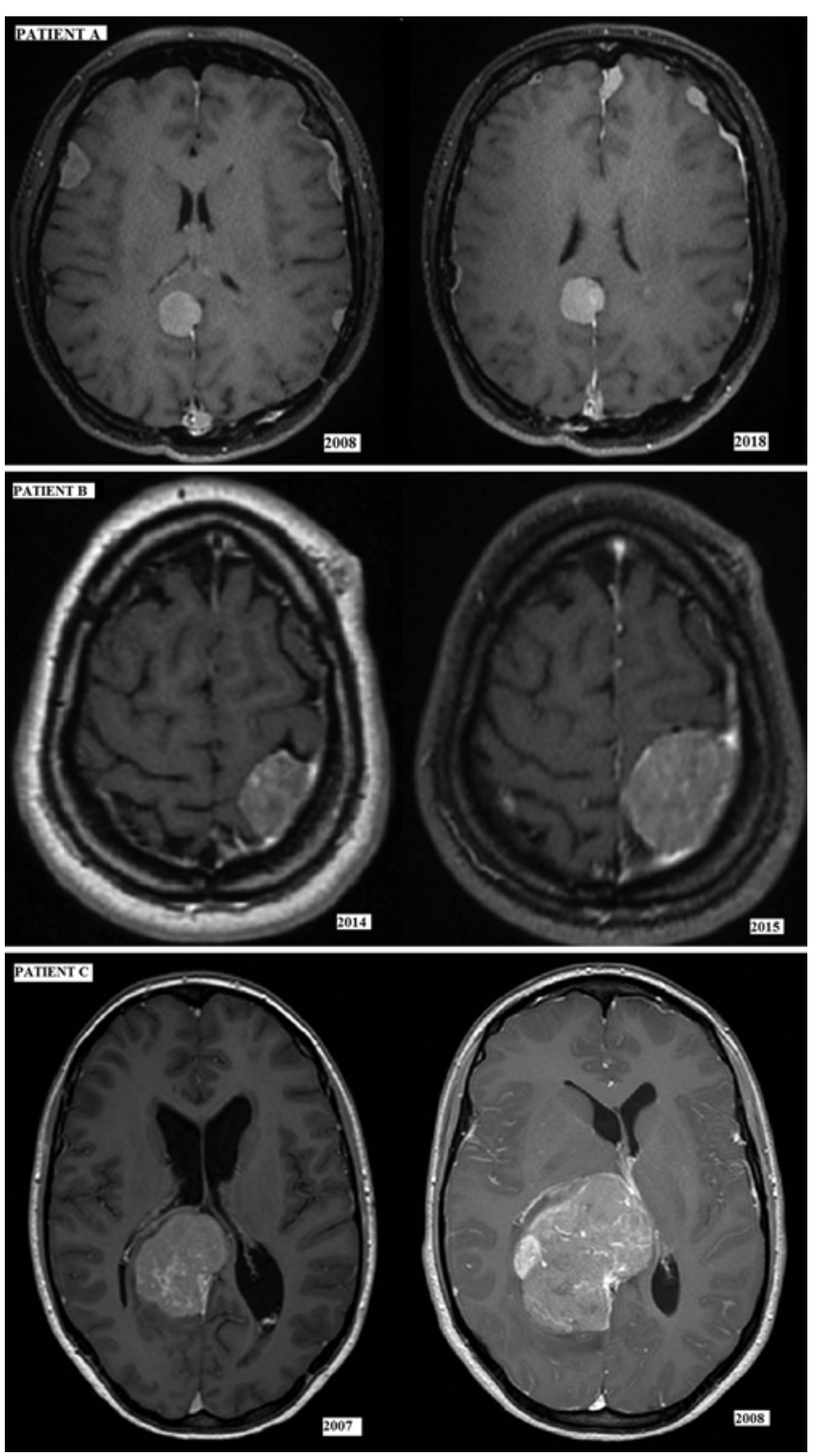

FIG. 3. Clinical applications of the scoring system. Patient A: initial score $=0$ (tumor diameter $<2.5 \mathrm{~cm}=0$, presence of calcifications $=0$, absence of peritumoral edema $=0$, hypointense on T2W MRI $=0$ ), low risk of rapid growth, confirmed annual growth rate $0.3 \mathrm{~cm}^{3} / \mathrm{yr}$. Patient B: initial score $=4$ (tumor diameter $<2.5 \mathrm{~cm}=0$, absence of calcifications $=2$, absence of peritumoral edema $=0$, hyperintense on T2W MRI $=2$ ), intermediate risk of rapid growth, confirmed annual growth rate 16.21 $\mathrm{cm}^{3} / \mathrm{yr}$. Patient C: initial score $=10$ (tumor diameter $\geq 4 \mathrm{~cm}=6$, absence of calcifications $=2$, absence of peritumoral edema $=0$, hyperintense on $\mathrm{T} 2 \mathrm{~W} M R \mathrm{I}=2$ ), high risk of rapid growth, confirmed annual growth rate $72.342 \mathrm{~cm}^{3} / \mathrm{yr}$.

It was previously established that the genetic severity score correlates with the presence of meningiomas. ${ }^{15}$ We did not find a correlation between the genetic severity score and rapid meningioma growth. Considering that multiple meningiomas in the same patient have various growth rates, this suggests that the NF2 mutation is necessary for tumor initiation, but additional somatic events at a genomic, expression, or epigenetic level participate in tumor growth or explain the variations of tumor growth rates within a single patient. This could mean that the genetic severity score predisposes to the presence of multiple meningiomas but does not determine the growth patterns of the individual meningiomas.

When compared with patients with sporadic meningiomas, patients with NF2-related meningiomas are significantly younger, and their mean tumor volume at diagnosis is significantly smaller. ${ }^{11,17}$ However, the rates of slow versus rapid tumor growth are not statistically significantly different between NF2-related meningiomas (83.2\% NF2 meningiomas with slow growth vs $16.8 \%$ NF2 meningiomas with rapid growth) and sporadic meningiomas $(78.7 \%$ vs $21.2 \%) .^{11,17}$ This is a further argument to support the notion that NF2 predisposes to the development of multiple meningiomas that then have independent growth rates.

The growth patterns of meningiomas in our cohort of NF2 patients are compatible with those in the literature. ${ }^{4,5,18}$ More often than not, they present with a slow linear growth pattern and seldom require therapeutic interventions. Thus in our center, we tend to recommend an initial observation for asymptomatic meningiomas in NF2 patients. ${ }^{19}$ When treatment is warranted, surgery is considered as first-line therapy, ${ }^{3,5,18,19}$ as findings from our study and from the literature show that NF2-associated meningiomas occur more frequently in surgically accessible locations.

In our series, the meningiomas in NF2 patients did not seem to have a more aggressive histopathological grade when compared with sporadic ones. Among our resected meningiomas, 25 were grade I and 26 were grade II. We think that this almost-50/50 distribution is a selection bias because usually meningiomas that undergo surgery are those that exhibit rapid growth or are symptomatic, and some of our grade I meningiomas were "en route" meningiomas removed during surgeries for other meningiomas or schwannomas. This contradicts other published series where NF2-associated meningiomas were found to be histologically more aggressive than sporadic ones. ${ }^{20}$

Finally, when comparing the slow-growth versus the rapid-growth group, we noticed that most of the rapidgrowth tumors that underwent surgery were a histopathological grade II, while most of the slow-growth tumors were a grade I. This is compatible with previous results ${ }^{5}$ and the general assumption that stable or slow-growing tumors are grade I. It contradicts previous findings where limited evidence was found in favor of the correlation of volumetric growth rates with histological diagnosis. ${ }^{21}$

In our study, only 2 meningiomas were successfully treated using Gamma Knife radiosurgery (GKRS) because they were inaccessible to surgery: one was located in the cavernous sinus and the other in the jugular foramen with a markedly slowed growth rate after GKRS. In the 5 other cases in which radiotherapy was used, results were rather disappointing. One patient underwent CyberKnife radiosurgery for a convexity meningioma. He experienced continued rapid growth later and eventually developed a neurological deficit that warranted surgery 1 year later. Four patients received conformational radiotherapy $(\mathrm{n}=$ $2)$, proton therapy $(n=1)$, or conformational radiotherapy followed by CyberKnife radiosurgery $(n=1)$ as adjuvant 
treatments postoperatively, with treatment failure and recurrence of all 4 meningiomas with rapid growth rates.

Since the AIMSS score seems valid in NF2-associated meningiomas, in particular in smaller meningiomas with a relatively high growth risk, it might seem legitimate to suggest a proactive treatment using radiosurgery in those specific cases. In fact, there are no definitive arguments against the use of fractioned external-beam radiotherapy or stereotactic radiosurgery in NF2 patients. A study in 2009 claimed good control with radiosurgery of 49 meningiomas in 12 patients. ${ }^{22}$ These meningiomas were claimed to be "asymptomatic but showed growth on serial imaging and were in eloquent locations in which further growth could lead to morbidity." A more recent study treated 12 patients for a total of 87 meningiomas with GKRS:23 $25 \%$ of procedures resulted in mild toxicity, $5 \%$ of the therapies had immediate local failures, and $67 \%$ resulted in distant treatment failures. Four of the 12 patients died during follow-up, and the authors stated that the cause of death was "progressive meningiomas." Based on these results, we would not conclude that proactive treatment with GKRS is better than simple observation in NF2 patients with multiple meningiomas. However, other papers have shown that radiosurgery could be an effective alternative for NF2-associated meningiomas especially in challenging cases. ${ }^{24,25}$ It is reported to be an effective treatment in up to $96 \%$ of cases, with manageable side effects and no malignant transformations. ${ }^{24}$ The main concern remains radiation-induced secondary tumors in the treatment field, malignant transformations, and acceleration of growth following stereotactic radiosurgery. ${ }^{26,27}$

\section{Study Limitations}

The current study is a retrospective analysis of NF2 patients from a national referral center. A prospective study needs to be performed with long-term follow-up for improved understanding of the natural history of intracranial meningiomas.

\section{Conclusions}

This study shows that the risk factors for rapid growth of meningiomas in NF2 patients are a larger tumor volume on diagnosis, presence of peritumoral edema, absence of calcifications, and hyperintense or isointense signal on T2W MRI. The AIMSS score that integrates these risk factors seems to provide a good estimate of the overall risk of rapid tumor growth in meningiomas in NF2 patients. This can aid in evaluating and counseling NF2 patients regarding prognosis and timely management of their intracranial meningiomas.

\section{Acknowledgments}

We thank Mrs. Véronique Pieters for her help in obtaining the medical records and Dr. Bruno Law Ye for his help with the Carestream software.

\section{References}

1. Evans DG, Howard E, Giblin C, et al. Birth incidence and prevalence of tumor-prone syndromes: estimates from a UK family genetic register service. Am J Med Genet A. 2010;152A(2):327-332.

2. Evans DG, Sainio M, Baser ME. Neurofibromatosis type 2. J Med Genet. 2000;37(12):897-904.

3. Asthagiri AR, Parry DM, Butman JA, et al. Neurofibromatosis type 2. Lancet. 2009;373(9679):19741986.

4. Aboukais R, Zairi F, Baroncini M, et al. Intracranial meningiomas and neurofibromatosis type 2. Acta Neurochir (Wien). 2013;155(6):997-1001.

5. Goutagny S, Bah AB, Henin D, et al. Long-term follow-up of 287 meningiomas in neurofibromatosis type 2 patients: clinical, radiological, and molecular features. Neuro Oncol. 2012;14(8):1090-1096.

6. Baser ME, Friedman JM, Aeschliman D, et al. Predictors of the risk of mortality in neurofibromatosis 2. Am J Hum Genet. 2002;71(4):715-723.

7. Dirks MS, Butman JA, Kim HJ, et al. Long-term natural history of neurofibromatosis Type 2-associated intracranial tumors. J Neurosurg. 2012;117(1):109-117.

8. Evers S, Verbaan D, Sanchez E, Peerdeman S. 3D Volumetric measurement of neurofibromatosis type 2-associated meningiomas: association between tumor location and growth rate. World Neurosurg. 2015;84(4):1062-1069.

9. Lawson McLean AC, Rosahl SK. Growth dynamics of intracranial tumors in patients with neurofibromatosis type 2 . World Neurosurg. 2017;98:152-161.

10. Hashimoto N, Rabo CS, Okita Y, et al. Slower growth of skull base meningiomas compared with non-skull base meningiomas based on volumetric and biological studies. $J$ Neurosurg. 2012;116(3):574-580.

11. Lee EJ, Kim JH, Park ES, et al. A novel weighted scoring system for estimating the risk of rapid growth in untreated intracranial meningiomas. J Neurosurg. 2017;127(5):971-980.

12. Evans DG, Huson SM, Donnai D, et al. A clinical study of type 2 neurofibromatosis. Q J Med. 1992;84(304):603-618.

13. Evans DGR. Neurofibromatosis type 2 (NF2): a clinical and molecular review. Orphanet J Rare Dis. 2009;4:16.

14. Ferner RE, Shaw A, Evans DG, et al. Longitudinal evaluation of quality of life in 288 patients with neurofibromatosis $2 . J$ Neurol. 2014;261(5):963-969.

15. Halliday D, Emmanouil B, Pretorius P, et al. Genetic Severity Score predicts clinical phenotype in NF2. J Med Genet. 2017;54(10):657-664.

16. Parry DM, Eldridge R, Kaiser-Kupfer MI, et al. Neurofibromatosis 2 (NF2): clinical characteristics of 63 affected individuals and clinical evidence for heterogeneity. Am J Med Genet. 1994;52(4):450-461.

17. Brugada-Bellsolà F, Teixidor Rodríguez $\mathrm{P}$, RodríguezHernández A, et al. Growth prediction in asymptomatic meningiomas: the utility of the AIMSS score. Acta Neurochir (Wien). 2019;161(11):2233-2240.

18. Blakeley JO, Evans DG, Adler J, et al. Consensus recommendations for current treatments and accelerating clinical trials for patients with neurofibromatosis type 2. Am J Med Genet A. 2012;158A(1):24-41.

19. Goutagny S, Kalamarides M. Meningiomas and neurofibromatosis. J Neurooncol. 2010;99(3):341-347.

20. Perry A, Giannini C, Raghavan R, et al. Aggressive phenotypic and genotypic features in pediatric and NF2associated meningiomas: a clinicopathologic study of 53 cases. J Neuropathol Exp Neurol. 2001;60(10):994-1003.

21. Fountain DM, Soon WC, Matys T, et al. Volumetric growth rates of meningioma and its correlation with histological diagnosis and clinical outcome: a systematic review. Acta Neurochir (Wien). 2017;159(3):435-445.

22. Wentworth S, Pinn M, Bourland JD, et al. Clinical experience with radiation therapy in the management of 
neurofibromatosis-associated central nervous system tumors. Int J Radiat Oncol Biol Phys. 2009;73(1):208-213.

23. Liu A, Kuhn EN, Lucas JT Jr, et al. Gamma Knife radiosurgery for meningiomas in patients with neurofibromatosis Type 2. J Neurosurg. 2015;122(3):536-542.

24. Birckhead B, Sio TT, Pollock BE, et al. Gamma Knife radiosurgery for neurofibromatosis type 2-associated meningiomas: a 22-year patient series. J Neurooncol. 2016;130(3):553-560.

25. Nguyen T, Chung LK, Sheppard JP, et al. Surgery versus stereotactic radiosurgery for the treatment of multiple meningiomas in neurofibromatosis type 2: illustrative case and systematic review. Neurosurg Rev. 2019;42(1):85-96.

26. Baser ME, Evans DG, Jackler RK, et al. Neurofibromatosis 2 , radiosurgery and malignant nervous system tumours. $\mathrm{Br} J$ Cancer. 2000;82(4):998.

27. Evans DGR, Birch JM, Ramsden RT, et al. Malignant transformation and new primary tumours after therapeutic radiation for benign disease: substantial risks in certain tumour prone syndromes. J Med Genet. 2006;43(4):289-294.

\section{Disclosures}

The authors report no conflict of interest concerning the materials or methods used in this study or the findings specified in this paper.

\section{Author Contributions}

Conception and design: Kalamarides, Abi Jaoude. Acquisition of data: Abi Jaoude, Goutagny. Analysis and interpretation of data: Abi Jaoude. Drafting the article: Abi Jaoude. Critically revising the article: Kalamarides, Peyre. Reviewed submitted version of manuscript: Kalamarides, Abi Jaoude, Peyre, Goutagny, Parfait. Approved the final version of the manuscript on behalf of all authors: Kalamarides. Statistical analysis: Degos. Study supervision: Kalamarides. Genetic testing: Parfait.

\section{Supplemental Information}

Online-Only Content

Supplemental material is available with the online version of the article.

Supplemental Figs. 1-3. https://thejns.org/doi/suppl/10.3171/ 2020.3.JNS192382.

\section{Correspondence}

Michel Kalamarides: Hôpital de la Pitié Salpêtrière, Paris, France. michel.kalamarides@aphp.fr. 